\title{
Controllability for a class of parallelly connected polynomial systems
}

\author{
Dragan Nešić \\ Department of Electrical and Electronic Engineering, \\ The University of Melbourne, \\ Parkville, 3052, Victoria, Australia \\ ph $+61+3+93445357$, fax $+61+3+93446678$ \\ d.nesic@ee.mu.oz.au
}

Keywords: complete controllability, discrete-time, null controllability, nonlinear, polynomial systems.

\begin{abstract}
Null controllability for a class of parallelly connected discrete-time polynomial systems is considered. We prove for this class of systems that a necessary and sufficient condition for null controllability of the parallel connection is that all its subsystems are null controllable. Consequently, the controllability test splits into a number of easy-to-check tests for the subsystems. The test for complete controllability is also presented and it is subtly different from the null controllability test. A similar statement is then given for complete controllability of a class of parallelly connected continuous-time polynomial systems. The result is somewhat unexpected when compared to the classical linear systems result. We identify the phenomenon which shows the difference between the linear and nonlinear cases.
\end{abstract}

\section{Introduction}

Controllability represents one of the most fundamental notions in control theory since it characterizes basic limitations to the performance of a plant. The importance of controllability stems from its intimate relation to the important concept of a minimal realization and the stabilizability problem. Hence, characterization of controllability properties of a controlled system should be an important part of any thorough control analysis or synthesis.

An important class of nonlinear models are polynomial models, which can be used to model a wide variety of plants and processes. Polynomial discrete-time models are used in an approach to black box identification of nonlinear systems [HaKe, HaUn]. In this paper, we consider a class of parallelly connected polynomial models, which are called URYSON models [HaKe, HaUn]. An important subclass of this class of models are generalized Hammerstein models, which consist of parallelly connected linear and nonlinear systems [HaKe, HaUn]. Despite their very simple structure, these models often turn out to be appropriate to model some practically important plants. For example, the models of a cement mill [Ke] and a cooling water circulation of a thermal power plant [BaIs] were identified in generalized Hammerstein form.

Controllability for classes of polynomial discrete-time systems has been addressed in [EvMu1, EvMu3, EvMu2, NMBM1, NMBM2, NeMa1, Ne1, NeMa2, NMBM3]. Papers [EvMu1] and [EvMu3] provide necessary and sufficient conditions for complete controllability of two classes of bilinear systems. In [EvMu2], conditions for complete controllability for linear systems with positive controls were presented. Notice that linear systems with positive controls can be regarded as a class of polynomial systems with square input nonlinearity. In [NMBM1] we addressed state null controllability of scalar polynomial systems of the form $x_{k+1}=f\left(x_{k}, u_{k}\right), x, u \in \mathbb{R}$ and a generic controllability result was proved. A generalization was presented in [NMBM2, NMBM3] where we obtained necessary and sufficient conditions for output dead-beat controllability of a class of planar odd polynomial systems given by the I/O equation $y_{k+1}=f\left(y_{k}, u_{k-1}, u_{k}\right)$, where $y \in \mathbb{R}$ and $u \in \mathbb{R}$ are respectively the output and input of the system. Symbolic computation approaches for null controllability problem of general polynomial systems were investigated in [NeMa1, NeMa2] and a solution based on the QEPCAD software package was obtained 
for general polynomial systems with rational coefficients. The results of [NeMa1, NeMa2] are applicable to the class of polynomial systems considered in this paper. However, large computational requirements decrease the efficiency of these methods and certain structural assumptions are needed to increase their applicability. In [Ne1] we considered a class of generalized Hammerstein systems, where we obtained an easy-to-check analytic null controllability test. We proved that the overall system is null (completely) controllable if and only if its subsystems are null (completely) controllable. This is a surprising result since it is known for parallel connections of linear systems that controllability of subsystems does not always guarantee controllability of the overall system [Ka] (the poles of the subsystems must not coincide).

In this paper we generalize results from [Ne1] to multiple parallel connections with arbitrary monomial input nonlinearities. We also prove a complete controllability result and an equivalent result for continuous time systems. The phenomena which show differences between the linear and nonlinear cases are identified and analyzed. An interesting interpretation of our results is that by "non-linearizing" an uncontrollable linear system we may recover controllability (more precise statements are given below). Related results for controllability and observability for series connections of linear blocks and static monomial nonlinearities (simple Wiener-Hammerstein systems) can be respectively found in [Ne2] and [Ne3].

The outline of the paper is as follows. After presenting preliminaries, in Section 3 we present background results which we need in the sequel and provide motivation for the problem we consider. In Section 4 we present a proof for null and complete controllability of a single parallel connection. This result is used in Section 5 as a building block to prove more general results on null and complete controllability of URYSON models (multiple parallel connections). Complete controllability of continuous-time parallelly connected systems is treated in Section 6 and we summarize our results in the last section.

\section{Preliminaries}

Sets of real, natural, rational and complex numbers are respectively denoted as $\mathbb{R}, \mathbb{N}, \mathbb{Q}$ and $\mathbb{C}$. Consider polynomial discrete-time systems of the following form:

$$
\begin{aligned}
S_{1}: x_{1}(k+1) & =F_{1} x_{1}(k)+g_{1} u^{q_{1}}(k) \\
S_{2}: x_{2}(k+1)= & F_{2} x_{2}(k)+g_{2} u^{q_{2}}(k) \\
S_{3}: x_{3}(k+1)= & F_{3} x_{3}(k)+g_{3} u^{q_{3}}(k) \\
\cdots & \cdots \\
S_{m}: x_{m}(k+1)= & F_{m} x_{m}(k)+g_{m} u^{q_{m}}(k)
\end{aligned}
$$

where $x_{i} \in \mathbb{R}^{n_{i}}, \sum_{i} n_{i}=n, u \in \mathbb{R}, F_{i} \in \mathbb{R}^{n_{i} \times n_{i}}, g_{i} \in \mathbb{R}^{n_{i} \times 1}, q_{i}>0, q_{i} \in \mathbb{N}, \forall i$ and $q_{i} \neq q_{j}, \forall i \neq j$.

We denote a sequence of controls $\{u(0), u(1), \ldots\}$ as $U$ where $u(i) \in \mathbb{R}$ and its truncation of length $N$, that is $\{u(0), \ldots, u(N-1)\}$, as $U_{N}$. The state of the system (1) at a time step $N$, which is obtained when a sequence $U_{N}$ is applied to the system and which emanates from the initial state $x(0)$, is denoted as $x\left(N, x(0), U_{N}\right)$. We give below the definitions that are used in the sequel:

Definition 1 The system (1) is null controllable if for any initial state $x(0) \in \mathbb{R}^{n}$ there exists a positive integer $N=N(x(0))$ and a control sequence $U_{N}$ such that $x\left(N, x(0), U_{N}\right)=0$.

Definition 2 If in Definition 1 there exists a fixed integer $\bar{N} \in \mathbb{N}$ such that $\forall x(0)$ it holds that $N(x(0))<$ $\bar{N}$, then we say that there exists a uniform bound on the dead-beat time.

Definition 3 The system (1) is completely controllable if $\forall x(0), x_{f} \in \mathbb{R}^{n}$ there exists a positive integer $N=N\left(x(0), x_{f}\right)$ and $U_{N}$ such that $x\left(N, x(0), U_{N}\right)=x_{f}$.

The characteristic polynomial of a matrix $F_{i}$ is denoted as $p_{F_{i}}(\lambda)=\operatorname{det}\left(\lambda I-F_{i}\right)$. Given a polynomial:

$$
p(\lambda)=\lambda^{t}+a_{t-1} \lambda^{t-1}+\ldots+a_{1} \lambda+a_{0}
$$

we denote the degree of $p$ as $\operatorname{deg}(p(\lambda))=t$. We introduce a new polynomial $p^{[q]}(\lambda)$ which is obtained from $p(\lambda)$ when all the coefficients $a_{i}$ are taken with a power $q>0, q \in \mathbb{Q}$, that is we write:

$$
p^{[q]}(\lambda)=\lambda^{t}+a_{t-1}^{q} \lambda^{t-1}+\ldots+a_{1}^{q} \lambda+a_{0}^{q}
$$


For $q=\frac{l_{1}}{l_{2}}, l_{i} \in \mathbb{N}, l_{i}>0$ where $l_{2}$ is even, we would always make sure that $a_{i} \geq 0$, so that if $p(\lambda)$ has real coefficients, then $p^{[q]}(\lambda)$ also has real coefficients. If we are given a polynomial $H=\lambda^{s}+h_{s-1} \lambda^{s-1}+$ $\ldots+h_{1} \lambda+h_{0}$, we use the notation:

$$
(p \cdot H)^{[q]}(\lambda)=\lambda^{t+s}+\left(h_{s-1}+a_{t-1}\right)^{q} \lambda^{t+s-1}+\ldots+\left(h_{1} a_{0}+h_{0} a_{1}\right)^{q} \lambda+\left(a_{0} h_{0}\right)^{q}
$$

If $q \in \mathbb{N}$, we can use repeatedly the binomial formula $(a+b)^{q}=\sum_{j=0}^{q}\left(\begin{array}{c}q \\ j\end{array}\right) a^{q-j} b^{j}$, where $\left(\begin{array}{c}q \\ j\end{array}\right)=$ $\frac{q !}{j !(q-j) !}$, to find the coefficients of $(p \cdot H)^{[q]}(\lambda)$. Hence, the polynomial $(p \cdot H)^{[q]}(\lambda)$ is obtained when we first multiply the polynomials $p$ and $H$ and then take $q$ th powers of all the coefficients of the product polynomial. Notice that the following holds: $(p \cdot H)^{[q]}(\lambda)=(H \cdot p)^{[q]}(\lambda) ;\left(p^{\left[q_{1}\right]}(\lambda)\right)^{\left[q_{2}\right]}=p^{\left[q_{1} q_{2}\right]}(\lambda)$; and $p^{[1]}(\lambda)=p(\lambda)$.

Definition 4 [CLO] A greatest common divisor of polynomials $p_{1}, p_{2}$ is a polynomial $h$ such that: $h$ divides $p_{1}$ and $p_{2}$; if $p$ is another polynomial which divides $p_{1}$ and $p_{2}$, then $p$ divides $h$. When $h$ has these properties we write $h=G C D\left(p_{1}, p_{2}\right)$.

Definition 5 Given a polynomial $p_{1}$ with roots $\left\{\sigma_{1}, \ldots, \sigma_{n_{1}}\right\}$ and polynomial $p_{2}$ with roots $\left\{\zeta_{1}, \ldots, \zeta_{n_{2}}\right\}$, their resultant is denoted as $\operatorname{Res}\left(p_{1}, p_{2}\right)$ and is defined as

$$
\operatorname{Res}\left(p_{1}, p_{2}\right)=\prod_{i, j}\left(\sigma_{i}-\zeta_{j}\right)
$$

Notice that two polynomials have a common root if and only if their resultant is equal to zero. Resultant of two polynomials can be obtained as a function of coefficients of the polynomials by using the Sylvester matrix [CLO].

Given a set of vectors $B_{i} \in \mathbb{R}^{n \times 1}, i=1,2, \ldots, f$, their span is denoted as:

$$
\operatorname{span}\left\{B_{1}, \ldots, B_{f}\right\}=\left\{x: x=\sum_{i=1}^{f} B_{i} \alpha_{i}, \alpha_{i} \in \mathbb{R}\right\}
$$

and their positive span is denoted as

$$
\operatorname{span}_{+}\left\{B_{1}, \ldots, B_{f}\right\}=\left\{x: x=\sum_{i=1}^{f} B_{i} \alpha_{i}, \alpha_{i} \geq 0\right\}
$$

Theorem 1 [EvMu1, Ev] Suppose that a matrix A has no real strictly positive or zero eigenvalues. Then there exists a polynomial $c(\lambda)=\sum_{i=0}^{T} c_{i} \lambda^{i}, c_{i}>0$ such that $c(A)=0$.

Theorem 2 [EvMu1] Consider a set of vectors $A^{i} b, i=0, \ldots, r$. If there exist $\alpha_{i}>0$ such that the following condition is satisfied

$$
\sum_{i=0}^{r} \alpha_{i} A^{i} b=0
$$

then $\operatorname{span}_{+}\left\{A^{r} b, \ldots, A b, b\right\}=\operatorname{span}\left\{A^{r} b, \ldots, A b, b\right\}$.

Comment 1 A special form of Theorem 2 can be interpreted in a geometric way, which is more suitable for our purposes. In Theorem 2 suppose that $r>n$ and that the first $n$ vectors $A^{i} b \in \mathbb{R}^{n}, i=0,1, \ldots, n-1$ are linearly independent. The cone generated by these vectors is denoted as $C=s p_{+}\left\{A^{n-1} b, \ldots, b\right\}$. Then obviously a positive linear combination of the $r-n$ remaining vectors $A^{i} b, i=n, \ldots, r$ must be in the interior of the negative cone $C^{-}=s p_{+}\left\{-A^{n-1} b, \ldots,-b\right\}$ in order for the condition (2) to hold.

It is not difficult to verify that this holds in general and we can state the following: Consider $n$ linearly independent vectors $A^{k_{n-1}} b, \ldots, A^{k_{1}} b, b \in \mathbb{R}^{n}$ with $k_{i+1}>k_{i}$. Denote the cone which is generated by these vectors as $C=s p_{+}\left\{A^{k_{n-1}} b, \ldots, A^{k_{1}} b, b\right\}$. The cone $C$ has a non-empty interior in $\mathbb{R}^{n}$. If there exists a vector $A^{k_{n}} b$ such that it belongs to the interior of the negative cone $C^{-}$, then the positive span of the vectors $s p_{+}\left\{A^{k_{n}} b, A^{k_{n-1}} b, \ldots, A^{k_{1}} b, b\right\}$ is the whole space $\mathbb{R}^{n}$. Notice that this implies that there exist positive numbers $\alpha_{i}$ such that

$$
\sum_{i=0}^{n} \alpha_{i} A^{k_{i}} b=0, \quad k_{i+1}>k_{i}, k_{i} \in \mathbb{N}
$$




\section{Motivation and background}

Testing controllability of general nonlinear systems is known to be a computationally very hard problem [So]. Hence, it is of utmost importance to identify classes of nonlinear systems which are relevant in control theory and whose structure can be used to facilitate controllability testing by reducing the complexity of the controllability test. We show in the sequel that systems (1) offer such a possibility.

The main idea exploited in this article is to view the system (1) as a parallel connection of simple Hammerstein systems $S_{i}, i=1,2, \ldots, m$ and to link the controllability properties of the subsystems $S_{i}$ with the controllability of their parallel connection (1). We note that null and complete controllability tests for subsystems $S_{i}$ are known from the literature. Indeed, consider a subsystem $S_{i}$ of $(1)$ with the input monomial nonlinearity $u^{q_{i}}$. Then we have:

Theorem $3 S_{i}$ is completely (null) controllable if and only if:

1. $[K a]$ for $q_{i}=2 t+1, t \in \mathbb{N}$

(a) $\operatorname{rank}\left[\lambda I-F_{i}: g_{i}\right]=n_{i}, \forall \lambda \in \mathbb{C}(\forall \lambda \in \mathbb{C}-\{0\})$

2. [EvMu1, Ne1] for $q_{i}=2 t, t>0, t \in \mathbb{N}$

(a) $\operatorname{rank}\left[\lambda I-F_{i}: g_{i}\right]=n_{i}, \forall \lambda \in \mathbb{C}(\forall \lambda \in \mathbb{C}-\{0\})$

(b) $F_{i}$ has no real strictly positive or zero eigenvalues (no real strictly positive eigenvalues)

Indeed, if $q_{i}$ is and odd integer, we need to use standard linear complete/null controllability tests from [Ka]. On the other hand, if $q_{i}$ is even, we view the system $S_{i}$ as a linear system with positive controls, for which complete and null controllability conditions can be found in [EvMu1, Ne1].

It is rather obvious that complete (null) controllability of the subsystems $S_{i}$ of the system (1) is necessary for complete (null) controllability of the overall system (1). However, it is a well known fact that for linear control systems this is not sufficient [Ka]. Consider the system (1) and suppose that $m=2, q_{1}=q_{2}=1$, that is we have a parallel connection of two linear systems. Denote the sets of eigenvalues of the matrices $F_{i}$ as $\mathcal{P}_{i}$, and then we can state

Theorem $4[\mathrm{Ka}]$ A parallel connection of two linear systems $S_{1}$ and $S_{2}$ is completely (null) controllable if and only if

1. both subsystems $S_{1}$ and $S_{2}$ are completely (null) controllable, and

2. $\mathcal{P}_{1} \cap \mathcal{P}_{2}=\emptyset\left(\nexists \sigma \neq 0, \sigma \in \mathcal{P}_{1} \cap \mathcal{P}_{2}\right)$.

We refer to Condition 2 of Theorem 4 hereafter as an interconnection condition. Hence, in the linear case, besides the obvious necessary controllability Condition 1 of Theorem 4, we also need the extra interconnection condition. The focus of our article is on characterizing similar interconnection conditions for complete/null controllability of systems (1), which lead to necessary and sufficient complete/null controllability conditions for (1). The phenomenon which shows the subtle difference between linear and nonlinear cases is illustrated by the following example.

Example 1 Consider the following linear system, which is a parallel connection of two scalar null controllable subsystems:

$$
\begin{aligned}
& x_{1}(k+1)=x_{1}(k)+u(k) \\
& x_{2}(k+1)=x_{2}(k)+u(k)
\end{aligned}
$$

The system (4) is not null controllable since for $\lambda=1$ we have that rank $[\lambda I-A: b]=1$ (the interconnection condition is violated since subsystems have a common non-zero pole). Suppose now that we insert a cubic nonlinearity at the input of the second subsystem, and hence we obtain the new system:

$$
\begin{array}{ll}
S_{1}: & x_{1}(k+1)=x_{1}(k)+u(k) \\
S_{2}: & x_{2}(k+1)=x_{2}(k)+u^{3}(k)
\end{array}
$$


Both subsystems $S_{1}$ and $S_{2}$ are obviously null controllable, but it is not yet clear whether their parallel connection is. To investigate this, consider the state of the system (5) at the time $k=2$. Since $S_{1}$ is null controllable we assume without loss of generality that $x_{1}(0)=0$ and we have:

$$
\begin{aligned}
& x_{1}(2)=u(0)+u(1) \\
& x_{2}(2)=x_{2}(0)+u^{3}(0)+u^{3}(1)
\end{aligned}
$$

If we want to render $x_{1}(2)=0$ by choice of $u(0), u(1)$, then we have that $u(0)=v$ and $u(1)=-v$, where $v \in \mathbb{R}$ is arbitrary. However, this choice of controls leads to cancellation of $v$ in the second equation in (6) and hence $x_{2}(2)$ can not be in general rendered zero. Nevertheless, let us consider the state of the system (5) at time step $k=3\left(x_{1}(0)=0\right.$ is assumed as before). We have then that:

$$
\begin{aligned}
& x_{1}(3)=u(0)+u(1)+u(2) \\
& x_{2}(3)=x_{2}(0)+u^{3}(0)+u^{3}(1)+u^{3}(2)
\end{aligned}
$$

There are many choices of $u(0), u(1), u(2)$ which render $x_{1}(3)=0$. One such choice is $u(0)=2 v, u(1)=$ $-v, u(2)=-v$, where $v \in \mathbb{R}$ is arbitrary. With this control sequence we obtain that $x_{2}(3)=x_{2}(0)+6 v^{3}$ and if we chose $v=\left(-x_{2}(0) / 6\right)^{1 / 3}$, we have that $x_{1}(3)=0, x_{2}(3)=0$ for arbitrary initial conditions, which means that the system (5) is null controllable.

Example can be interpreted as follows: by non-linearizing the input to one subsystem of a linear parallel connection which is not null controllable, we can recover null controllability. In a sense, the cubic nonlinearity destroyed the linear interconnection condition (Condition 2, Theorem 4), due to which the linear parallel connection was not null controllable. We show below that the situation in our example always happens for nonlinear systems (1).

\section{A single parallel connection}

In this section, we concentrate on systems (1) for which $m=2$. First, main results of the section are stated and commented on. Relationships and differences between our results and the known linear results of Theorem 4 are explained and an interpretation of our results stated. In the second part of the section we prove several technical lemmas and main theorems of this section.

Consider a parallel connection of two simple Hammerstein systems with the input nonlinearities of the form $u^{q_{i}}$ :

$$
\begin{aligned}
& S_{1}: x_{1}(k+1)=F_{1} x_{1}(k)+g_{1} u^{q_{1}}(k) \\
& S_{2}: x_{2}(k+1)=F_{2} x_{2}(k)+g_{2} u^{q_{2}}(k)
\end{aligned}
$$

where $x_{1} \in \mathbb{R}^{n_{1}}, x_{2} \in \mathbb{R}^{n_{2}}, u \in \mathbb{R}, q_{i} \in \mathbb{N}, q_{i}>0, q_{1} \neq q_{2}$ and the matrices $F_{i}, g_{i}$ have the appropriate dimensions.

The main results of this section are stated below:

Theorem 5 The system (8) is null controllable if and only if its both subsystems $S_{1}$ and $S_{2}$ are null controllable.

Hence, if we consider the system (8), there is no interconnection condition which could lead to loss of null controllability if the subsystems are null controllable. This is the main difference between the parallel connection of purely linear systems (see Theorem 4) and the nonlinear system (8). As a result, the null controllability test for the system (8) is very simple and it splits into two tests for the subsystems, which are stated in Theorem 3. Hence, the phenomenon illustrated in Example 1 always happens and we can state that: if a parallel connection of two linear null controllable systems is not null controllable, by inserting an odd monomial nonlinearity in front of one subsystem we recover null controllability of the parallel connection. Note that if we wanted to insert an even nonlinearity in front of a subsystem with the same purpose, the corresponding subsystem should not have any real strictly positive eigenvalues (see Theorem 3).

In order to state the complete controllability result, we introduce the following notation: the sets of eigenvalues of matrices $F_{i}$ are denoted as $\mathcal{P}_{i}, i=1,2$.

Theorem 6 The system (8) is completely controllable if and only if 
1. the subsystems $S_{1}$ and $S_{2}$ are completely controllable, and

2. $0 \notin \mathcal{P}_{1} \cap \mathcal{P}_{2}$.

Therefore, for complete controllability we have an interconnection condition but notice the subtle difference with linear case (Condition 2 in Theorem 4). Obviously, the interconnection condition for the nonlinear system (8) is less restrictive than the corresponding linear one. Complete controllability test for (8) is again rather simple (use Theorems 3 and 6) and only linear algebra is needed to test it. The statement of Theorem 6 can be further simplified if at least one of the matrices $F_{i}, i=1,2$ is non-singular, in which case Condition 2 of the theorem always holds:

Corollary 1 Suppose that at least one of the matrices $F_{i}, i=1,2$ is non-singular. The system (8) is completely controllable if and only if both subsystems $S_{1}$ and $S_{2}$ are completely controllable.

We emphasize that in Theorem 6 the interconnection condition (Condition 2) is implied by Condition 1 as soon as at least one of $q_{i}$ is an even integer. Indeed, from Theorem 3 it follows that if $q_{i}$ is even, the corresponding matrix $F_{i}$ should be non-singular for the subsystem $S_{i}$ to be completely controllable. Therefore, we have:

Corollary 2 Suppose that at least one of $q_{i}, i=1,2$ is an even integer. The system (8) is completely controllable if and only if both subsystems $S_{1}$ and $S_{2}$ are completely controllable.

Hence, Condition 2 of Theorem 6 is not implied by Condition 1 only when both $q_{1}, q_{2}$ are odd integers. Consequently, the interconnection condition from Theorem 6 is always satisfied for the class of systems considered in [Ne1], where we had $q_{1}=1, q_{2}=2$. In this sense, in Theorem 6 we identified a genuinely new phenomenon, which can occur only when both $q_{i}, i=1,2$ are odd.

Finally, the difference in statements of Theorems 5 and 6 can be illustrated by the following trivial system

$$
\begin{aligned}
& x_{1}(k+1)=u(k) \\
& x_{2}(k+1)=u^{3}(k)
\end{aligned}
$$

whose both scalar subsystems are completely controllable (and hence null controllable). The system (9) is obviously null controllable but it is not completely controllable since the final states $x_{f}$ that are not in the set $\left\{x:-x_{1}^{3}+x_{2}=0\right\}$ can not be reached from any initial state $x(0) \in \mathbb{R}^{2}$.

In the rest of this section we prove the needed technical lemmas and Theorems 5 and 6 . Results of Lemmas are used to construct special control sequences which are used in proofs of Theorems 5 and 6 , and which are very similar to the one used in Example 1.

Lemma 1 Suppose that a pair of matrices $A \in \mathbb{R}^{n \times n}$ and $b \in \mathbb{R}^{n \times 1}$ is controllable and $A$ is non-singular. Then, given any positive integer $T \in \mathbb{N}$, there exist a set of positive integers of the form:

$$
\begin{aligned}
k_{0} & =0 \\
k_{i+1} & \geq k_{i}+T, i=0,1, \ldots, n-1
\end{aligned}
$$

such that

$$
\operatorname{span}\left[A^{k_{n-1}} b: A^{k_{n-2}} b: \ldots: A^{k_{1}} b: b\right]=\mathbb{R}^{n}
$$

In other words, there exist integers of the form (10) such that $\operatorname{rank}\left[A^{k_{n-1}} b: A^{k_{n-2}} b: \ldots: A^{k_{1}} b: b\right]=n$. Proof of Lemma 1: Because of controllability of $A, b$ and non-singularity of $A$ we can write:

$$
\operatorname{rank} A^{k}\left[A^{n-1} b: \ldots: b\right]=n, \forall k \in \mathbb{N}
$$

Pick an integer $s_{1} \geq T$. If $A^{s_{1}} b$ and $b$ are linearly independent, let $k_{1}=s_{1}$. Suppose that the vectors $A^{s_{1}} b$ and $b$ are linearly dependent. Hence, there exists $e_{1} \in \mathbb{R}$ such that $A^{s_{1}} b=e_{1} b$. Consider now the vector $A^{s_{1}+1} b$ and $b$ and suppose that they are linearly dependent. That implies that $A^{s_{1}} b$ and $A^{s_{1}+1} b$ are also linearly dependent, which contradicts (12). Hence, we can let $k_{1}=s_{1}+1$. The construction of the remaining $k_{i}$ is carried out in the same manner by considering the linearly independent vectors $A^{k_{i-1}} b, \ldots, b$ and a new vector $A^{s_{i}} b$ with $s_{i} \geq k_{i-1}+T$, which proves Lemma 1. Q.E.D. 
Lemma 2 Consider polynomials

$$
\begin{aligned}
& p_{1}(\lambda)=\lambda^{n_{1}}+b_{n_{1}-1} \lambda^{n_{1}-1}+\ldots+b_{1} \lambda+b_{0}, b_{i} \in \mathbb{R}, b_{0} \neq 0 \\
& p_{2}(\lambda)=\lambda^{n_{2}}+a_{n_{2}-1} \lambda^{n_{2}-1}+\ldots+a_{1} \lambda+a_{0}, a_{i} \in \mathbb{R}, a_{0} \neq 0
\end{aligned}
$$

There exists a polynomial $H(\lambda)$ with real coefficients of the degree at most $n_{2}$ such that the polynomials $p_{1}(\lambda)$ and $\left(p_{2} \cdot H\right)^{\left[\frac{q_{2}}{q_{1}}\right]}(\lambda), q_{i} \in \mathbb{N}, q_{i}>0$ have no common roots if and only if $q_{1} \neq q_{2}$.

Note: if $q_{1}$ is even, then both $p_{2}$ and $H$ are assumed to have non-negative real coefficients.

Proof of Lemma 2: Necessity: Suppose that $q_{1}=q_{2}$. Obviously, $p_{2}^{\left[\frac{q_{2}}{q_{1}}\right]}(\lambda)=p_{2}(\lambda)$. Then, if $G C D\left(p_{1}, p_{2}\right) \neq 1$ we have for any polynomial $H$ that $G C D\left(p_{1}, H p_{2}\right) \neq 1$, which proves necessity.

Sufficiency: Sufficiency is proved by construction of polynomial $H$ satisfying conditions of the lemma. Suppose that $q_{1} \neq q_{2}$. Denote the set of roots of the polynomial $p_{1}(\lambda)$ as $\Sigma=\left\{\sigma_{1}, \sigma_{2}, \ldots, \sigma_{n_{1}}\right\}$.

Notice that the resultant of $p_{1}(\lambda)$ and $\left(H \cdot p_{2}\right)^{\left[\frac{q_{2}}{q_{1}}\right]}(\lambda)$ can be written as:

$$
\operatorname{Res}\left(p_{1}(\lambda),\left(H \cdot p_{2}\right)^{\left[\frac{q_{2}}{q_{1}}\right]}(\lambda)\right)=\prod_{i=1}^{n_{1}}\left(H \cdot p_{2}\right)^{\left[\frac{q_{2}}{q_{1}}\right]}\left(\sigma_{i}\right)
$$

We introduce the following polynomial

$$
H(\lambda)=\lambda^{n_{2}}+h
$$

and compute the polynomial $\left(p_{2} \cdot H\right)^{\left[\frac{q_{2}}{q_{1}}\right]}(\lambda)$ :

$$
\left(p_{2} \cdot H\right)^{\left[\frac{q_{2}}{q_{1}}\right]}(\lambda)=\lambda^{2 n_{2}}+a_{n_{2}-1}^{\frac{q_{2}}{q_{1}}} \lambda^{2 n_{2}-1}+\ldots+\left(a_{0}+h\right)^{\frac{q_{2}}{q_{1}}} \lambda^{n_{2}}+\ldots+\left(h a_{1}\right)^{\frac{q_{2}}{q_{1}}} \lambda+\left(a_{0} h\right)^{\frac{q_{2}}{q_{1}}}
$$

The only way for the polynomial (15) to fail in proving Lemma 2 is that there exists a root $\sigma_{j} \in \Sigma$ of $p_{1}(\lambda)$ for which the polynomial $\left(p_{2} \cdot H\right)^{\left[\frac{q_{2}}{q_{1}}\right]}\left(\sigma_{j}\right) \equiv 0$ (see the expression $(14)$ ). In other words, the polynomial (16) evaluated at $\lambda=\sigma_{j}$ is trivial when viewed as a polynomial in $h$.

Suppose that there exists $\sigma_{j} \in \Sigma$ for which $\left(p_{2} \cdot H\right)^{\left[\frac{q_{2}}{q_{1}}\right]}\left(\sigma_{j}\right) \equiv 0$. If we introduce the new variable $t=h^{\frac{1}{q_{1}}}$, we can write:

$$
\underbrace{\sigma_{j}^{2 n_{2}}+\ldots+a_{1}^{\frac{q_{2}}{q_{1}}} \sigma_{j}^{n_{2}+1}}_{C_{1}}+\left(a_{0}+t^{q_{1}}\right)^{\frac{q_{2}}{q_{1}}} \sigma_{j}^{n_{2}}+\underbrace{\left(a_{n_{2}-1}^{\frac{q_{2}}{q_{1}}} \sigma_{j}^{n_{2}-1}+\ldots+a_{0}^{\frac{q_{2}}{q_{1}}}\right)}_{C_{2}} t^{q_{2}}=0, \forall t \in \mathbb{R}
$$

In other words, we have that (since $b_{0} \neq 0$ then $\sigma_{j} \neq 0$ ) the following must hold:

$$
\left(a_{0}+t^{q_{1}}\right)^{q_{2}} \equiv\left(-C_{1}-C_{2} t^{q_{2}}\right)^{q_{1}} \sigma_{j}^{-n_{2} \cdot q_{1}}
$$

Consider first the situation $q_{2}>q_{1}$. Since $a_{0} \neq 0$, the polynomial on the L.H.S. of (18) is always a weighted sum of monomials of the form $t^{j q_{1}}, j=0,1, \ldots, q_{2}$. Hence, the polynomial on the L.H.S. consists of $q_{2}+1$ monomials. On the other hand, for any $C_{1}, C_{2}, \sigma_{j}$ the polynomial on the R.H.S. of (18) is a weighted sum of at most $q_{1}+1$ monomials of the form $t^{i q_{2}}, i=0,1,2, \ldots, q_{1}$. However, since $q_{2}>q_{1}$ the polynomial on the L.H.S. contains always more monomials than the polynomial on the R.H.S. and hence we have a contradiction.

Consider now the situation $q_{2}<q_{1}$ and suppose that (18) holds. The polynomial on the L.H.S. always has $q_{2}+1 \geq 2$ monomials since $a_{0} \neq 0$. If $C_{1}=0$ and/or $C_{2}=0$, then the polynomial on the R.H.S. has one monomial only, a contradiction. If, on the other hand, $C_{1} \neq 0, C_{2} \neq 0$ then the polynomial on the R.H.S. has $q_{1}+1$ monomials and since $q_{1}>q_{2}$ we have again a contradiction.

Therefore, (18) can never hold if $q_{1} \neq q_{2}$, which proves the lemma. Q.E.D.

Comment 2 We remark that the condition $a_{0} \neq 0$ and $b_{0} \neq 0$ is crucial for Lemma 2 to work. Indeed, suppose that $p_{1}$ and $p_{2}^{\left[\frac{q_{2}}{\left.q_{1}\right]}\right.}$ have a common root equal to zero. Then for any $H$ with real coefficients we have that $\left(H \cdot p_{2}\right)^{\left[\frac{q_{2}}{q_{1}}\right]}(0)=0$ and hence Res $\left(p_{1},\left(H \cdot p_{2}\right)^{\left[\frac{q_{2}}{q_{1}}\right]}\right)=0$ for any polynomial $H$ with real coefficients. 
Comment 3 We need the following observation in the sequel. Suppose that for an arbitrary $r \in \mathbb{N}, r>1$ we introduce a polynomial $p_{2}=p_{2}\left(\lambda^{r}\right)$, deg $\left(p_{2}\right)=n_{2} \cdot r$. If we want to apply Lemma 2 to an arbitrary polynomial $p_{1}$ and the polynomial $p_{2}$ in the given form, we can use straightforwardly the method from Lemma 2. The polynomial $H=\lambda^{n_{2} \cdot r}+h$ constructed using Lemma 2 is also a polynomial in $\lambda^{r}$. Hence, the product $p_{2} \cdot H=\left(p_{2} \cdot H\right)\left(\lambda^{r}\right)$ is a polynomial in $\lambda^{r}$. This shows that for an arbitrary polynomial $p_{2}$ in $\lambda^{r}$, we can find a polynomial $H$ so that $p_{2} \cdot H$ satisfies conditions of Lemma 2 and is a polynomial in $\lambda^{r}$.

Comment 4 Lemma 2 is instrumental in the proof of the main result and we present its special case which better illustrates the nonlinear mechanism which will be shown later to destroy the linear interconnection condition (see Example 1).

Let us consider system (8) with $q_{1}=1$ and $q_{2}=q$ and $F_{i}$ are non-singular. Denote $p_{1}(\lambda)=p_{F_{2}}(\lambda)$ and $p_{2}(\lambda)=p_{F_{1}}(\lambda)$. Assume that $p_{F_{1}}^{[q]}\left(\sigma_{j}\right)=0$ for some $\sigma_{j}$, an eigenvalue of $F_{2}$. Introduce again the polynomial $H=\lambda^{n_{1}}+h$ and consider

$$
\left(p_{F_{1}} \cdot H\right)^{[q]}(\lambda)=\lambda^{2 n_{1}}+a_{n_{1}-1}^{q} \lambda^{2 n_{1}-1}+\ldots+a_{1}^{q} \lambda^{n_{1}+1}+\left(a_{0}+h\right)^{q} \lambda^{n_{1}}+h^{q}\left(a_{n_{1}-1}^{q} \lambda^{n_{1}-1}+\ldots+a_{0}^{q}\right)
$$

From equation (14), we see that the polynomial $H$ fails in proving the result of Lemma 2 if and only if for some root of $p_{F_{2}}(\lambda)$, denoted as $\sigma_{j}$, we have that $\left(p_{F_{1}} \cdot H\right)^{[q]}\left(\sigma_{j}\right)$ is a trivial polynomial in $h$.

Consider now the eigenvalues $\sigma_{i}$ of $F_{2}$ for which $p_{F_{1}}^{[q]}\left(\sigma_{i}\right) \neq 0$ we obtain

$$
\left(p_{F_{1}} \cdot H\right)^{[q]}\left(\sigma_{i}\right)=p_{F_{1}}^{[q]}\left(\sigma_{i}\right) h^{q}+\ldots
$$

which is a non-trivial polynomial in $h$. If, on the other hand, we consider the eigenvalue $\sigma_{j}$ of $F_{2}$ for which $p_{F_{1}}^{[q]}\left(\sigma_{j}\right)=0$, we obtain:

$$
\left(p_{F_{1}} \cdot H\right)^{[q]}\left(\sigma_{j}\right)=\left(\sum_{i=1}^{q-1}\left(\begin{array}{c}
q \\
i
\end{array}\right) a_{0}^{q-i} h^{i}\right) \sigma_{j}^{n_{1}}
$$

Since $F_{1}, F_{2}$ are assumed to be non-singular, both $a_{0}$ and $\sigma_{j}$ are not zero. It follows that the polynomial (20) is a non-trivial polynomial in $h$ only if $q>1$. Indeed, the sum on the R.H.S. of (20) is defined only if $q-1>0$. Formula (20) is crucial and we will show later that in the case $q_{2}>1$ we can use this property to recover null controllability, whereas for $q_{2}=1$ (purely linear case) it is impossible to do so. We postpone further details until the proof of the main result.

Lemma 3 Consider arbitrary polynomials $p_{1}(\lambda)=\sum_{i=0}^{n_{1}} \alpha_{i} \lambda^{i}$ and $p_{2}(\lambda)=\sum_{i=0}^{n_{2}} \beta_{i} \lambda^{i}$. Given any $r>$ $n_{2}, r \in \mathbb{N}$, we have that:

$$
\left(p_{1}\left(\lambda^{r}\right) \cdot p_{2}(\lambda)\right)^{[q]}=p_{1}^{[q]}\left(\lambda^{r}\right) p_{2}^{[q]}(\lambda)
$$

Proof of Lemma 3: By straightforward calculations we have that:

$$
p_{1}\left(\lambda^{r}\right) \cdot p_{2}(\lambda)=\left(\sum_{i=0}^{n_{1}} \alpha_{i} \lambda^{r i}\right)\left(\sum_{i=0}^{n_{2}} \beta_{i} \lambda^{i}\right)=\sum_{i=0}^{n_{1}} \sum_{j=0}^{n_{2}} \alpha_{i} \beta_{j} \lambda^{r i+j}
$$

and therefore we have that

$$
\begin{aligned}
\left(p_{1}\left(\lambda^{r}\right) \cdot p_{2}(\lambda)\right)^{[q]} & =\sum_{i=0}^{n_{1}} \sum_{j=0}^{n_{2}}\left(\alpha_{i} \beta_{j}\right)^{q} \lambda^{r i+j}=\sum_{i=0}^{n_{1}} \sum_{j=0}^{n_{2}} \alpha_{i}^{q} \beta_{j}^{q} \lambda^{r i+j}=\left(\sum_{i=0}^{n_{1}} \alpha_{i}^{q} \lambda^{r i}\right)\left(\sum_{j=0}^{n_{2}} \beta_{j}^{q} \lambda^{j}\right) \\
& =p_{1}^{[q]}\left(\lambda^{r}\right) p_{2}^{[q]}(\lambda) \quad \text { Q.E.D. }
\end{aligned}
$$

Lemma 4 Consider a matrix $F$, which has no real positive or zero eigenvalues. Given any integer $T \in \mathbb{N}$, there exists another integer $T_{1} \geq T$ such that $F^{T_{1}}$ has no real positive or zero eigenvalues.

Proof of Lemma 4: The eigenvalues of $F^{k}$ are $\lambda_{i}^{k}$, where $\lambda_{i}$ are eigenvalues of $F$. We can write $\lambda_{i}$ as

$$
\lambda_{i}=r_{i}\left(\cos \left(\phi_{i}\right)+j \sin \left(\phi_{i}\right)\right), j=\sqrt{-1}
$$


It is obvious then that:

$$
\lambda_{i}^{k}=r_{i}^{k}\left(\cos \left(k \phi_{i}\right)+j \sin \left(k \phi_{i}\right)\right)
$$

Consider now the values of $I=\left\{k^{*}: k^{*} \in \mathbb{N}, k^{*}>0\right.$, for which $F^{k^{*}}$ has positive or zero eigenvalues $\}$. It is easy to see that since $F$ has no zero eigenvalues then $F^{k}$ has no zero eigenvalues $\forall k \in \mathbb{N}$. If $F$ has real negative eigenvalues, the integers of the form $k^{*}=2 t, t \in \mathbb{N}, t>0$ belong to the critical set I. If $F$ has pure imaginary eigenvalues, then the integers of the form $k^{*}=4 t, t \in \mathbb{N}, t>0$ belong to $I$. Finally, if there are any complex eigenvalues $\lambda_{i}$, it is easy to see that only eigenvalues $\lambda_{i}$ for which $\phi_{i}$ has the form $l \pi, l \in \mathbb{Q}$ are such that $\exists k^{*}$ so that $\lambda_{i}^{k^{*}} \in \mathbb{R}, \lambda_{i}^{k^{*}}>0$. We can write for any such eigenvalue that $\phi_{i}= \pm \frac{d_{i}}{m_{i}} \pi, m_{i}, d_{i} \in \mathbb{N}, d_{i}<m_{i}$ and $G C D\left(m_{i}, d_{i}\right)=1$. Then the integers of the form $k^{*}=t m_{i}, t \in \mathbb{N}, t>0$ if $d_{i}$ is even and $k^{*}=2 t m_{i}$ if $d_{i}$ is odd also belong to the critical set $I$. Since $F$ has finitely many eigenvalues, the set of critical integers $I$ has finitely many generating numbers, that is for any $k^{*} \in I$, we can write $k^{*}=2 t$ or $t m_{i}$ or $2 t m_{i}$ for $t \in \mathbb{N}, t>0$. Since $F$ has no positive eigenvalues, finitely many prime numbers are contained in the set $I$. Consequently, given any $T \in \mathbb{N}$, any prime number $P$ not in the set $I$ which is larger than $T$ satisfies the conditions of Lemma 4 and we can let $T_{1}=P$. Q.E.D.

Comment 5 In the proof of Theorem 5 we assume without loss of generality that the matrices $F_{1}$ and $F_{2}$ are non-singular. Zero modes can be ignored when investigating null controllability. To see this, suppose that there are some zero eigenvalues of $F_{1}$ and $F_{2}$. By using a non-singular linear coordinate transformation we can write the system in new coordinates:

$$
\begin{aligned}
& \left(\begin{array}{l}
\xi_{1}^{1}(k+1) \\
\xi_{1}^{2}(k+1)
\end{array}\right)=\left(\begin{array}{cc}
F_{11} & 0 \\
0 & J_{1}
\end{array}\right)\left(\begin{array}{l}
\xi_{1}^{1}(k) \\
\xi_{1}^{2}(k)
\end{array}\right)+\left(\begin{array}{l}
g_{1}^{1} \\
g_{1}^{2}
\end{array}\right) u^{q_{1}}(k) \\
& \left(\begin{array}{l}
\xi_{2}^{1}(k+1) \\
\xi_{2}^{2}(k+1)
\end{array}\right)=\left(\begin{array}{cc}
F_{22} & 0 \\
0 & J_{2}
\end{array}\right)\left(\begin{array}{l}
\xi_{2}^{1}(k) \\
\xi_{2}^{2}(k)
\end{array}\right)+\left(\begin{array}{l}
g_{2}^{1} \\
g_{2}^{2}
\end{array}\right) u^{q_{2}}(k)
\end{aligned}
$$

where $F_{11}, F_{22}$ are non-singular and $J_{1}, J_{2}$ are nilpotent matrices. Suppose that for any $\xi_{1}^{1}(0), \xi_{2}^{1}(0)$ we can find a control sequence $U_{N}$ which yields $\xi_{1}^{1}\left(N, \xi_{1}^{1}(0), U_{N}\right)=0$ and $\xi_{2}^{1}\left(N, \xi_{2}^{1}(0), U_{N}\right)=0$. Then, by concatenating this control sequence with a sequence of $u(k)=0, k=N, N+1, \ldots$, there exists time $T$ such that $\xi(T)=0$, because $J_{1}, J_{2}$ are nilpotent. Hence, we can concentrate on zeroing only the states corresponding to non-zero modes when proving null controllability. Consequently (see Theorem 3), both subsystems $S_{1}$ and $S_{2}$ can be assumed completely controllable when proving null controllability.

Proof of Theorem 5: Necessity part of the proof is obvious and we concentrate only on sufficiency. We assume without loss of generality that matrices $F_{1}$ and $F_{2}$ are non-singular (see Comment 5) and subsystems $S_{1}$ and $S_{2}$ are completely controllable.

Given an arbitrary polynomial $p(\lambda)=\lambda^{T}+\alpha_{T-1} \lambda^{T-1}+\ldots+\alpha_{0}$, we introduce the following control sequence, which is denoted as $U[p(\lambda)]$ :

$$
\begin{aligned}
u(0)= & v\left(n_{2}\right) \\
u(1)= & \alpha_{T-1}^{\frac{1}{q_{1}}} v\left(n_{2}\right) \\
u(2)= & \alpha_{T-2}^{\frac{1}{q_{1}}} v\left(n_{2}\right) \\
\cdots= & \cdots \\
u(T)= & \alpha_{0}^{\frac{1}{q_{1}}} v\left(n_{2}\right) \\
u\left(n_{1}+j_{0}\right)= & 0,0<j_{0}<k_{1}-T, k_{1}>T, j_{0} \in \mathbb{N} \\
u\left(k_{1}\right)= & v\left(n_{2}-1\right) \\
u\left(k_{1}+1\right)= & \alpha_{T-1}^{\frac{1}{q_{1}}} v\left(n_{2}-1\right) \\
u\left(k_{1}+2\right)= & \alpha_{T-2}^{\frac{1}{q_{1}}} v\left(n_{2}-1\right) \\
\cdots= & \cdots \\
u\left(k_{1}+T\right)= & \alpha_{0}^{\frac{1}{q_{1}}} v\left(n_{2}-1\right) \\
u\left(k_{1}+T+j_{1}\right)= & 0,0<j_{1}<k_{2}-k_{1}-T, k_{2}>k_{1}+T, j_{1} \in \mathbb{N} \\
u\left(k_{2}\right)= & v\left(n_{2}-2\right)
\end{aligned}
$$




$$
\begin{array}{rll}
\ldots & \cdots \\
u\left(k_{n_{2}-1}\right)= & v(1) \\
u\left(k_{n_{2}-1}+1\right)= & \alpha_{T-1}^{\frac{1}{q_{1}}} v(1) \\
\cdots & \cdots \\
u\left(k_{n_{2}-1}+T\right)= & \alpha_{0}^{\frac{1}{q_{1}}} v(1)
\end{array}
$$

where $\alpha_{i}$ are the coefficients of the polynomial $p(\lambda)$ and $k_{i+1}>k_{i}+T$, where $T=\operatorname{deg}(p(\lambda))$, is a set of integers which we show how to chose below.

Theorem 5 is proved by construction and we distinguish several cases:

First case: Both $q_{1}$ and $q_{2}$ in (8) are odd integers. Since the subsystem $S_{1}$ is completely controllable, we assume without loss of generality that $x_{1}(0)=0$ (for any $\xi_{0} \in \mathbb{R}^{n_{1}}$ we can find a control sequence $U_{N}$ which yields $x_{1}\left(N, \xi_{0}, U_{N}\right)=0$ and let formally $x_{1}(0)=x_{1}\left(N, \xi_{0}, U_{N}\right)=0$ and $x_{2}(0)$ arbitrary - see Example 1).

Since $F_{1}$ and $F_{2}$ are assumed non-singular, their characteristic polynomials $p_{F_{1}}(\lambda)$ and $p_{F_{2}}(\lambda)$ satisfy conditions of Lemma 2 and it follows that we can find a polynomial $H(\lambda)$ such that $\operatorname{Res}\left(\left(p_{F_{1}}\right.\right.$. $\left.H)^{\left[\frac{q_{2}}{q_{1}}\right]}(\lambda), p_{F_{2}}(\lambda)\right) \neq 0$. Notice that this implies that the matrix $\left(p_{F_{1}} \cdot H\right)^{\left[\frac{q_{2}}{q_{1}}\right]}\left(F_{2}\right)$ is non-singular. With any such polynomial $H$, we apply the control sequence $U\left[p_{F_{1}}(\lambda) \cdot H(\lambda)\right]$ given in (26) to the system (8). Notice that the degree of the polynomial $p_{F_{1}}(\lambda) \cdot H(\lambda)$ is $2 n_{1}$ and at time step $k_{n_{2}-1}+2 n_{1}+1$ we have:

$$
\begin{aligned}
x_{1}\left(k_{n_{2}-1}+2 n_{1}+1\right) & =F_{2}^{k_{n_{2}-1}+2 n_{1}+1} \underbrace{x_{1}(0)}_{=0}+\underbrace{p_{F_{1}}\left(F_{1}\right)}_{=0} H\left(F_{1}\right)\left[F_{1}^{k_{n_{2}-1}} g_{1}: \ldots: F_{1}^{k_{1}} g_{1}: g_{1}\right]\left[v^{q_{1}}\left(n_{2}\right) \ldots v^{q_{1}}(1)\right]^{T} \\
& =0 \\
x_{2}\left(k_{n_{2}-1}+2 n_{1}+1\right) & =F_{2}^{k_{n_{2}-1}+2 n_{1}+1} x_{2}(0)+\underbrace{\left(p_{F_{1}} \cdot H\right)^{\left[\frac{q_{2}}{q_{1}}\right]}\left(F_{2}\right)}_{\text {non-singular }}\left[F_{2}^{k_{n_{2}-1}} g_{2}: \ldots: F_{2}^{k_{1}} g_{2}: g_{2}\right]\left[v^{q_{2}}\left(n_{2}\right) \ldots v^{q_{2}}(1)\right]^{T} \\
& =F_{2}^{k_{n_{2}-1}+2 n_{1}+1} x_{2}(0)+L\left[v^{q_{2}}\left(n_{2}\right) \ldots v^{q_{2}}(1)\right]^{T}
\end{aligned}
$$

The control sequence is chosen in such a way that it annihilates in the first state equation irrespective of the values $v(i)$ and integers $k_{i}$. Since we also assumed $x_{1}(0)=0$, we have that $x_{1}\left(k_{n_{2}-1}+2 n_{1}+1\right)=0$ irrespective of the values of $v(i), k_{i}$.

Next we show we can choose $v(i)$ and $k_{i}$ in order to render the state $x_{2}\left(k_{n_{2}-1}+2 n_{1}+1\right)=0 . \quad F_{2}$ is non-singular and $\left(F_{2}, g_{2}\right)$ controllable by our assumption and from Lemma 1 it follows that we can always choose $k_{i}, i=0,1, \ldots, n_{2}-1$ so that the vectors $F_{2}^{k_{i}} g_{2}$ are linearly independent. Suppose that we choose $k_{i}$ in such a way, then since $\left(p_{F_{1}} \cdot H\right)^{\left[\frac{q_{2}}{q_{1}}\right]}\left(F_{2}\right)$ is non-singular, the matrix $L$ in $(27)$ is non-singular. Finally, since $q_{2}$ is odd, we can always render $x_{2}\left(k_{n_{2}-1}+2 n_{1}+1\right)=0$ and the system is null controllable. Moreover, notice that since the image of the map $L\left[v^{q_{2}}\left(n_{2}\right) \ldots v^{q_{2}}(1)\right]^{T}, v(i) \in \mathbb{R}$ is $\mathbb{R}^{n_{2}}$, we can assign actually arbitrary $x_{2}\left(k_{n_{2}-1}+2 n_{1}+1\right) \in \mathbb{R}^{n_{2}}$.

Second case: One of $q_{i}$ is odd and another is even. Assume without loss of generality that $q_{2}$ is even with $q_{1}$ odd. In order to specify the control sequence that we use, we need to define several polynomials. As before from Lemma 2 it follows that there exists a polynomial $H_{1}(\lambda)$ such that $\operatorname{Res}\left(\left(p_{F_{1}}\right.\right.$. $\left.\left.H_{1}\right)^{\left[\frac{q_{2}}{q_{1}}\right]}(\lambda), p_{F_{2}}(\lambda)\right) \neq 0$ and consequently $\left(p_{F_{1}} \cdot H_{1}\right)^{\left[\frac{q_{2}}{q_{1}}\right]}\left(F_{2}\right)$ is non-singular (note that the degree of $\left(p_{F_{1}} \cdot H_{1}\right)^{\left[\frac{q_{2}}{q_{1}}\right]}(\lambda)$ is $\left.2 n_{1}\right)$.

Since the subsystem $S_{1}$ is completely controllable, we still assume that $x_{1}(0)=0$. Apply the control sequence $U\left[p_{F_{1}} \cdot H_{1}(\lambda)\right]$ where $H_{1}$ is chosen (using Lemma 2) so that $\left(p_{F_{1}} \cdot H_{1}\right)^{\left[q_{2}\right]}\left(F_{2}\right)$ is a non-singular matrix and the numbers $k_{i}$ are chosen (using Lemma 1) so that $F_{2}^{k_{i}} g_{2}$ are linearly independent. We need to concatenate this control sequence with another control sequence in order to complete the proof.

Since the subsystem $S_{2}$ is controllable, $\left(F_{2}, g_{2}\right)$ is a controllable pair and $F_{2}$ has no strictly positive or zero eigenvalues and this implies that there exists a polynomial with positive coefficients $c(\lambda)=\sum_{i=0}^{T_{1}} c_{i} \lambda^{i}$ such that $c\left(F_{2}\right)=0$ (See Theorem 1 ). From Theorem 2 it follows that given any $x^{*} \in \mathbb{R}^{n_{2}}$, there exist $\bar{c}_{i} \geq 0$ such that $\sum_{i=0}^{T_{1}} \bar{c}_{i} F_{2}^{i} g_{2}=x^{*}$. Introduce the polynomial

$$
\chi(\lambda)=\left(p_{F_{1}^{T_{1}+1}} \cdot H_{2}\right)^{\left[\frac{1}{q_{1}}\right]}\left(\lambda^{T_{1}+1}\right) \bar{c}^{\left[\frac{1}{q_{2}}\right]}(\lambda), T_{1}=\operatorname{deg}(\bar{c}(\lambda))
$$

where the polynomial $\bar{c}(\lambda)=\sum_{i=0}^{T_{1}} \bar{c}_{i} \lambda^{i}, \bar{c}_{i} \geq 0$ is to be chosen below; $p_{F_{1}^{T_{1}+1}}(\lambda)=\operatorname{det}\left(\lambda I-F_{1}^{T_{1}+1}\right)$; $\operatorname{deg}\left(p_{F_{1}^{T_{1}+1}}\left(\lambda^{T_{1}+1}\right)\right)=n_{1}\left(T_{1}+1\right) ; H_{2}=\lambda^{n_{1}\left(T_{1}+1\right)}+h_{2}$ is chosen (using Lemma 2$)$ so that $\left(p_{F_{1}^{T_{1}+1}}\right.$. 
$\left.H_{2}\right)^{\left[\frac{q_{2}}{q_{1}}\right]}\left(F_{2}^{T_{1}+1}\right)$ is non-singular. By using the notation $\chi(\lambda)=\sum_{i=0}^{R} \beta_{i} \lambda^{i}$, we apply the following control sequence to the system (8): the control sequence $U\left[p_{F_{1}} \cdot H_{1}(\lambda)\right]$ (see $(26)$ ) is concatenated by the control sequence $u\left(k_{n_{2}-1}+2 n_{1}+1+i\right)=\beta_{R-i} v(0), i=0,1, \ldots, R$. Under this control sequence, the state of the system at time step $r=k_{n_{2}}+2 n_{1}+R+2$ is:

$$
\begin{aligned}
x_{1}(r) & =F_{1}^{r} \underbrace{x_{1}(0)}_{=0}+F_{1}^{R+1} \underbrace{\left(p_{F_{1}} \cdot H_{1}\right)\left(F_{1}\right)}_{=0}\left[F_{1}^{k_{n_{2}-1}} g_{1}: \ldots: g_{1}\right]\left[v^{q_{1}}\left(n_{2}\right) \ldots v^{q_{1}}(1)\right]^{T}+\chi^{\left[q_{1}\right]}\left(F_{1}\right) g_{1} v^{q_{1}}(0) \\
x_{2}(r) & =F_{2}^{r} x_{2}(0)+\underbrace{F_{2}^{R+1}\left(p_{F_{1}} \cdot H_{1}\right)^{\left[\frac{q_{2}}{q_{1}}\right]}\left(F_{2}\right)}_{\text {non-singular }}\left[F_{2}^{k_{n_{2}-1}} g_{2}: \ldots: g_{2}\right]\left[v^{q_{2}}\left(n_{2}\right) \ldots v^{q_{2}}(1)\right]^{T}+\chi^{\left[q_{2}\right]}\left(F_{2}\right) g_{2} v^{q_{2}}(0) \\
& =F_{2}^{r} x_{2}(0)+\left[\hat{B}_{n_{2}}: \ldots: \hat{B}_{1}\right]\left[v^{q_{2}}\left(n_{2}\right) \ldots v^{q_{2}}(1)\right]^{T}+\hat{B}_{0} v^{q_{2}}(0)
\end{aligned}
$$

From Lemma 3 it follows that $\forall k \in \mathbb{N}$ we have

$$
\chi^{[k]}(\lambda)=\left(\left(p_{F_{1}^{T_{1}+1}} \cdot H_{2}\right)^{\left[\frac{1}{q_{1}}\right]}\left(\lambda^{T_{1}+1}\right) \bar{c}^{\left[\frac{1}{q_{2}}\right]}(\lambda)\right)^{[k]}=\left(p_{F_{1}^{T_{1}+1}} \cdot H_{2}\right)^{\left[\frac{k}{q_{1}}\right]}\left(\lambda^{T_{1}+1}\right) \bar{c}^{\left[\frac{k}{q_{2}}\right]}(\lambda)
$$

and hence we have that

$$
\begin{aligned}
& \chi^{\left[q_{1}\right]}\left(F_{1}\right)=\underbrace{\left(p_{F_{1}^{T_{1}+1}} \cdot H_{2}\right)^{\left[\frac{q_{1}}{q_{1}}\right]}\left(F_{1}^{T_{1}+1}\right)}_{=0} \bar{c}^{\left[\frac{q_{1}}{q_{2}}\right]}\left(F_{1}\right)=0 \\
& \chi^{\left[q_{2}\right]}\left(F_{2}\right)=\underbrace{\left(p_{F_{1} T_{1}+1} \cdot H_{2}\right)^{\left[\frac{q_{2}}{q_{1}}\right]}\left(F_{2}^{T_{1}+1}\right)}_{\text {non-singular }} \bar{c}^{\left[\frac{q_{2}}{q_{2}}\right]}\left(F_{2}\right)
\end{aligned}
$$

From (29) and (30) we obtain that $x_{1}(r)=0$ irrespective of $v(i), i=0,1, \ldots, n_{2}, \bar{c}(\lambda)$ and $k_{i}$. We use these degrees of freedom to show that by choosing $v(i), k_{i}$ and $\bar{c}(\lambda)$ we can render also $x_{2}(r)=0$.

Note that $\hat{B}_{i}, i=1,2, \ldots, n_{2}$ can be made linearly independent vectors (Lemma 1 ) so that the cone generated by them has a nonempty interior. As we already indicated, for arbitrary $x^{*} \in \mathbb{R}^{n_{2}}$ there exists $\bar{c}(\lambda)$ so that $\bar{c}\left(F_{2}\right) g_{2}=x^{*}$. Since $\left(p_{F_{1}^{T_{1}+1}} \cdot H_{2}\right)^{\left[\frac{q_{2}}{q_{1}}\right]}\left(F_{2}^{T_{1}+1}\right)$ is non-singular we can find the polynomial $\bar{c}(\lambda)$ so that $\hat{B}_{0}=\left(p_{F_{1}^{T_{1}+1}} \cdot H_{2}\right)^{\left[\frac{q_{2}}{q_{1}}\right]}\left(F_{2}^{T_{1}+1}\right) \bar{c}^{\left[\frac{q_{2}}{q_{2}}\right]}\left(F_{2}\right) g_{2}$ belongs to the interior of $s p_{+}\left\{-\hat{B}_{1}, \ldots,-\hat{B}_{n_{2}}\right\}$. With such a choice of $\bar{c}(\lambda)$ it follows that, $s p_{+}\left\{\hat{B}_{0}, \hat{B}_{1}, \ldots, \hat{B}_{n_{2}}\right\}=\operatorname{sp}\left\{\hat{B}_{0}, \hat{B}_{1}, \ldots, \hat{B}_{n_{2}}\right\}=\mathbb{R}^{n_{2}}$ since $\hat{B}_{i}, i=1,2, \ldots, n_{2}$ are linearly independent (see Theorem 2 and Comment 1 ), which completes the proof.

Case 3: Both $q_{1}$ and $q_{2}$ are even. We can use a very similar proof as in the previous case. The control sequence which we use in the proof is $U\left[c_{F_{1}} \cdot H_{1}(\lambda)\right]$ concatenated by the control sequence (see proof of

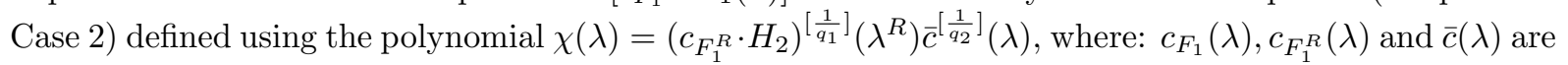
polynomials with positive and non-negative coefficients respectively such that $c_{F_{1}}\left(F_{1}\right)=0, c_{F_{1}^{R}}\left(F_{1}^{R}\right)=0$ and $\bar{c}(\lambda)$ is chosen in the same way as in the proof of Case $2 ; H_{1}$ and $H_{2}$ are polynomials with nonnegative coefficients, which are chosen so that $\left(c_{F_{1}} \cdot H_{1}\right)^{\left[\frac{q_{2}}{q_{1}}\right]}\left(F_{2}\right)$ and $\left(c_{F_{1}^{R}} \cdot H_{2}\right)^{\left[\frac{q_{2}}{q_{1}}\right]}\left(F_{2}^{R}\right)$ are non-singular (using Lemma 2); $R$ is an integer $R>T$, where $T$ is the degree of $c_{F_{1}}(\lambda)$, chosen so that $F_{1}^{R}$ has no real strictly positive or zero eigenvalues (see Lemma 4 ). The proof then follows word by word as in Case 2. Q.E.D.

Comment 6 Special control sequences that we used in the proof of Theorem 5 can not be used in the cases when $q_{1}=q_{2}$, which includes the linear case. Lemma 2 (see also Comment 4) states that it is always possible to construct the satisfactory control sequences used in the proof of our main result if and only if $q_{1} \neq q_{2}$. It is easy to show that if $q_{1}=q_{2}$, the parallel connection is null controllable if and only if the subsystems are null controllable and they also do not have common non-zero poles. Hence, for complete/null controllability in the case $q_{1}=q_{2}$ we need an interconnection condition and moreover the interconnection condition is completely equivalent to the one in the purely linear case $q_{1}=q_{2}=1$, given in Theorem 4 .

\section{Proof of Theorem 6:}

Necessity: Necessity of the first condition is obvious. To show that the second condition is necessary, suppose the subsystems $S_{1}, S_{2}$ are completely controllable and that the system (8) is completely controllable but that $0 \in \mathcal{P}_{1} \cap \mathcal{P}_{2}$. Notice that both $q_{1}, q_{2}$ should be odd in this case since if they are even, 
the existence of zero eigenvalues destroys complete controllability of subsystems. Hence, there exists a non-singular coordinate transformation so that the system (8) is in new coordinates given by (25). Since both subsystems are assumed controllable, we can assume that the pairs $\left(J_{1}, g_{1}^{2}\right)$ and $\left(J_{2}, g_{2}^{2}\right)$ in $(25)$ are both in controllability canonical form. If we denote the last entries of vectors $\xi_{1}^{2}(k)$ and $\xi_{2}^{2}(k)$ as $\eta_{1}(k)$ and $\eta_{2}(k)$ respectively, we see from $(25)$ that for any $k=0,1,2, \ldots$ it holds that:

$$
\begin{aligned}
& \eta_{1}(k+1)=u^{q_{1}}(k) \\
& \eta_{2}(k+1)=u^{q_{2}}(k)
\end{aligned}
$$

and hence if a final state $\xi_{f} \in \mathbb{R}^{n}$, which we want to reach, does not belong to the set $S_{C}=\{x$ : $\left.\left(\eta_{1}\right)^{q_{2}}-\left(\eta_{2}\right)^{q_{1}}=0\right\} \subset \mathbb{R}^{n}$, we can not reach it for any sequence of controls from any initial state $x(0) \in \mathbb{R}^{n}$ (see also the system (9)). This contradicts our assumption that the overall system is completely controllable.

Sufficiency: Suppose first that both subsystems $S_{1}$ and $S_{2}(8)$ are completely controllable and $F_{1}$ and $F_{2}$ are both non-singular. We can therefore assume that $x_{1}(0)$ can be arbitrarily preassigned using a pre-sequence of controls and we can think of $x_{1}(0)$ as a vector function of the control pre-sequence whose image is $\mathbb{R}^{n_{1}}$. From proof of Theorem 5 , we know that for any of the cases we presented there exists an integer $r$ and control sequence $U_{r}$ such that

$$
\begin{aligned}
& x_{1}(r)=F_{1}^{r} x_{1}(0) \\
& x_{2}(r)=F_{2}^{r} x_{2}(0)+L\left[v^{q_{2}}\left(n_{2}\right) \ldots v^{q_{2}}(1) v^{q_{2}}(0)\right]^{T}
\end{aligned}
$$

Hence, there exists a sequence of controls $U_{r}$, which is a function of $v(i)$, that has no effect on the first subsystem whereas the image of the map $L\left[v^{q_{2}}\left(n_{2}\right) \ldots v^{q_{2}}(1) v^{q_{2}}(0)\right]^{T}$ is $\mathbb{R}^{n_{2}}$. Bearing in mind that $F_{1}$ is non-singular and $x_{1}(0)$ can be preassigned arbitrarily, we see that given any $x_{1, f} \in \mathbb{R}^{n_{1}}$ and $x_{2, f} \in \mathbb{R}^{n_{2}}$, there exist $v(0), \ldots, v\left(n_{2}\right)$ and $x_{1}(0)$ (that is a pre-sequence of controls) so that $x_{1}(r)=x_{1, f}$ and $x_{2}(r)=x_{2, f}$.

Suppose now that both $S_{1}$ and $S_{2}$ are completely controllable but one of the matrices $F_{i}$, say $F_{1}$, is singular (in this case $q_{1}$ is necessarily odd). There exists a non-singular coordinate transformation such that the system (8) in new coordinates is:

$$
\begin{aligned}
\xi_{1}(k+1) & =F_{11} \xi_{1}(k)+g_{1}^{1} u^{q_{1}}(k) \\
\xi_{2}(k+1) & =J_{1} \xi_{2}(k)+g_{1}^{2} u^{q_{1}}(k) \\
x_{2}(k+1) & =F_{2} x_{2}(k)+g_{2} u^{q_{2}}(k)
\end{aligned}
$$

where $\left(F_{11}, g_{1}^{1}\right),\left(J_{1}, g_{2}^{1}\right),\left(F_{2}, g_{2}\right)$ are in controllability canonical form, $F_{11}, F_{2}$ are non-singular and $J_{1} \in \mathbb{R}^{r \times r}$ is nilpotent. In the same way as before, we think of $\xi_{1}(0)$ as a "control". We consider the non-zero modes of both subsystems $\left(\xi_{1}, x_{2}\right)$ and apply the control sequence $U_{R}$ which would have no effect on the $\xi_{1}$ subsystem, whereas arbitrary $x_{2, f}$ can be achieved with it and then concatenate this sequence with $r$ controls $u(R-1+i), i=1, \ldots, r$ to obtain:

$$
\begin{aligned}
\xi_{1}(R+r) & =F_{11}^{R+r} \xi_{1}(0)+\sum_{i=0}^{r-1} F_{11}^{r-1-i} g_{1}^{1} u^{q_{1}}(R+i) \\
\xi_{2}(R+r) & =\sum_{i=0}^{r-1} J_{1}^{r-1-i} g_{1}^{2} u^{q_{1}}(R+i) \\
x_{2}(R+r) & =F_{2}^{R+r} x_{2}(0)+F_{2}^{r} L\left[v^{q_{2}}\left(n_{2}\right) \ldots v^{q_{2}}(1) v^{q_{2}}(0)\right]^{T}+\sum_{i=0}^{r-1} F_{2}^{r-1-i} g_{2} u^{q_{2}}(R+i)
\end{aligned}
$$

First, since $\left(J_{1}, g_{1}^{2}\right)$ is controllable, given any $\xi_{2, f} \in \mathbb{R}^{r}$ we can choose $u(R+i), i=0,1, \ldots, r-1$ so that $\xi_{2}(R+r)=\xi_{2, f}$. Second, since $F_{11}$ is non-singular, given any $\xi_{1, f} \in \mathbb{R}^{n_{1}-r}$ we can pre-assign $\xi_{1}(0)$ so that $\xi_{1}(R+r)=\xi_{1, f}$. Finally, given any $x_{2, f} \in \mathbb{R}^{n_{2}}$, we can choose $v(0), \ldots, v\left(n_{2}\right)$ so that $x_{2}(R+r)=x_{2, f}$, which completes the proof. Q.E.D.

We close this section with an example which illustrates the method exploited in proofs of main results of the section. 
Example 2 Consider the system:

$$
\begin{aligned}
& S_{1}: x_{1}(k+1)=F_{1} x_{1}(k)+g_{1} u^{2}(k) \\
& S_{2}: x_{2}(k+1)=F_{2} x_{1}(k)+g_{2} u^{3}(k)
\end{aligned}
$$

where

$$
F_{1}=\left(\begin{array}{cc}
0 & 1 \\
-2 & 1
\end{array}\right), F_{2}=\left(\begin{array}{cc}
0 & 1 \\
-1 & -1
\end{array}\right), g_{1}=g_{2}=\left(\begin{array}{l}
0 \\
1
\end{array}\right)
$$

It is easy to verify that the system (35) is null and completely controllable using Theorems 5 and 6 . However, we use the approach exploited in the proofs of main results in order to illustrate our method. Notice that both subsystems $S_{1}$ and $S_{2}$ in (35) are null controllable since the pairs $\left(F_{1}, g_{1}\right)$ and $\left(F_{2}, g_{2}\right)$ are controllable (they are in controllability canonical form) and the matrix $F_{1}$ has no real strictly positive or zero eigenvalues. Indeed, the eigenvalues of $F_{1}$ are $0.5 \pm j 1.3229$. As a result, there exists a polynomial $c_{F_{1}}(\lambda)$ with positive coefficients such that $c_{F_{1}}\left(F_{1}\right)=0$. The polynomial $c_{F_{1}}(\lambda)=\lambda^{4}+\lambda^{3}+2 \lambda^{2}+2 \lambda+4$ is one such polynomial. To prove null controllability, since the subsystem $S_{1}$ is completely controllable, we can assume without loss of generality that $x_{1}(0)$ is a zero vector.

Assume also that the following control sequence is applied to the system:

$$
\begin{aligned}
& u(0)=v(1) ; u(1)=v(1) ; u(2)=\sqrt{2} v(1) ; u(3)=\sqrt{2} v(1) ; u(4)=2 v(1) ; \\
& u(5)=v(0) ; u(6)=v(0) ; u(7)=\sqrt{2} v(0) ; u(8)=\sqrt{2} v(0) ; u(9)=2 v(0) ;
\end{aligned}
$$

Then the systems equations at the time step $k=10$ are:

$$
\begin{aligned}
& x_{1}(10)=F_{1}^{10} \underbrace{x_{1}(0)}_{=0}+\underbrace{c_{F_{1}}^{\left[\frac{2}{2}\right]}\left(F_{1}\right)}_{=0}\left[F_{1}^{5} g_{1}: g_{1}\right]\left[v^{2}(1) v^{2}(0)\right]^{T} \\
& x_{2}(10)=F_{2}^{10} x_{2}(0)+\underbrace{c_{F_{1}}^{\left[\frac{3}{2}\right]}\left(F_{2}\right)}_{\text {non-singular non-singular }} \underbrace{\left[F_{2}^{5} g_{2}: g_{2}\right]}_{\text {non }}\left[v^{3}(1) v^{3}(0)\right]^{T}
\end{aligned}
$$

and since both matrices $c_{F_{1}}^{\left[\frac{3}{2}\right]}\left(F_{2}\right)$ and $\left[F_{1}^{5} g_{1}: g_{1}\right]$ are non-singular, the image of the mapping

$$
c_{F_{1}}^{\left[\frac{3}{2}\right]}\left(F_{2}\right)\left[F_{2}^{5} g_{2}: g_{2}\right]\left[v^{3}(1) v^{3}(0)\right]^{T}, v(0), v(1) \in \mathbb{R}
$$

is $\mathbb{R}^{2}$ and consequently the system is null controllable. Moreover, since the matrices $F_{1}$ and $F_{2}$ are both non-singular, the system is completely controllable. Indeed, suppose we want to transfer any initial state $\left(x_{1}^{T}(0) x_{2}^{T}(0)\right)^{T}$ to the state $\left(x_{1, f}^{T} x_{2, f}^{T}\right)^{T}$. Since the first system is completely controllable, we can assume without loss of generality that the initial state $x_{1}(0)$ was preassigned (using a pre-sequence of controls) so that $F_{1}^{10} x_{1}(0)=x_{1, f}$. The argument is then the same as before.

\section{Multiple parallel connections}

In this section we generalize the result on complete and null controllability to multiple parallel connections. The main results of the paper are given below:

Theorem 7 Consider a URYSON system (1). The system is null controllable if and only if all of its subsystems $S_{i}$ are null controllable.

Theorem 8 Consider a URYSON system (1). The system is completely controllable if and only if:

1. all of its subsystems $S_{i}$ are null controllable and

2. $\forall i, j \in\{1, \ldots, m\}, i \neq j$ we have that $0 \notin \mathcal{P}_{i} \cap \mathcal{P}_{j}$, where $\mathcal{P}_{i}$ denote the sets of eigenvalues of the matrices $F_{i}$.

From Theorems 7 and 8 we obtain very easy complete and null controllability tests for system (1). We concentrate below on proving only Theorem 7 since the methods from previous section can be used in a straightforward manner to prove Theorem 8.

An obvious consequence of Theorem 8 is: 
Corollary 3 Suppose that for system (1) at most one of $F_{i}, i=1,2, \ldots, m$ is a singular matrix. Then the system (1) is completely controllable if and only if all subsystems $S_{i}$ are completely controllable.

The following lemma generalizes the statement of Lemma 2 and is instrumental in proving the controllability result for multiple parallel connections:

Lemma 5 Consider a set of pairs $\left(F_{i}, q_{i}\right), i=1,2, \ldots, j$, where $F_{i} \in \mathbb{R}^{n_{1} \times n_{1}}$ are non-singular matrices; $q_{i} \in \mathbb{N}, q_{i}>0 ; q_{i} \neq q_{j}$ if $i \neq j$; and if $q_{i}$ is an even integer, the matrix $F_{i}$ has no real strictly positive eigenvalues. There exists a polynomial $\Pi_{j}(\lambda)$, which has no zero roots, such that

1. $\Pi_{j}^{\left[q_{i}\right]}\left(F_{i}\right)=0, \forall i=1,2, \ldots, j-1$, and

2. $\operatorname{Res}\left(p_{F_{j}}(\lambda), \Pi_{j}^{\left[q_{j}\right]}(\lambda)\right) \neq 0$

Proof of Lemma 5: Lemma is proved by construction of the polynomial $\Pi_{j}(\lambda)$. First, we use the notation $p_{F_{i}}(\lambda)$ for a characteristic polynomial of $F_{i}$ and $c_{F_{i}}(\lambda)$ for a polynomial with positive coefficients for which $c_{F_{i}}\left(F_{i}\right)=0$. If $q_{i}$ is even, the conditions of Lemma always guarantee the existence of $c_{F_{i}}(\lambda)$ - see Theorem 1. We introduce a set of polynomials $\psi_{j}, j=1,2, \ldots, j-1$, which are computed in the following way:

1. Polynomial $\psi_{1}(\lambda)$ :

(a) if $q_{1}$ is an odd integer, we let $r_{1}=1$ and $\psi_{1}\left(\lambda^{r_{1}}\right)=\psi_{1}(\lambda)=p_{F_{1}}(\lambda) \cdot H_{1}(\lambda)$, where $H_{1}$ is chosen using Lemma 2 so that $\operatorname{Res}\left(p_{F_{j}}(\lambda),\left(p_{F_{1}} \cdot H_{1}\right)^{\left[\frac{q_{j}}{q_{1}}\right]}(\lambda)\right) \neq 0$.

(b) if $q_{1}$ is an even integer, we let $r_{1}=1$ and $\psi_{1}\left(\lambda^{r_{1}}\right)=\psi_{1}(\lambda)=c_{F_{1}}(\lambda) \cdot H_{1}(\lambda)$, where $H_{1}$ is chosen using Lemma 2 so that $\operatorname{Res}\left(p_{F_{j}}(\lambda),\left(c_{F_{1}} \cdot H_{1}\right)^{\left[\frac{q_{j}}{q_{1}}\right]}(\lambda)\right) \neq 0$. We note that in this case $H_{1}$ is assumed to have non-negative coefficients.

2. Polynomial $\psi_{i}(\lambda), i=2, \ldots, j-1$ :

(a) if $q_{i}$ is an odd integer, we let $r_{i}>\operatorname{deg}\left(\psi_{i-1}\left(\lambda^{r_{i-1}}\right) \ldots \cdot \psi_{1}\left(\lambda^{r_{1}}\right)\right)$ and $\psi_{i}\left(\lambda^{r_{i}}\right)=p_{F_{i}^{r_{i}}}\left(\lambda^{r_{i}}\right) \cdot H_{i}\left(\lambda^{r_{i}}\right)$, where $H_{i}$ is chosen using Lemma 2 and Comment 3 so that $\operatorname{Res}\left(p_{F_{j}}(\lambda),\left(p_{F_{i}} \cdot H_{i}\right)^{\left[\frac{q_{j}}{q_{i}}\right]}\left(\lambda^{r_{i}}\right)\right) \neq 0$.

(b) if $q_{i}$ is an even integer, we let $r_{i}>\operatorname{deg}\left(\psi_{i-1}\left(\lambda^{r_{i-1}}\right) \cdot \ldots \cdot \psi_{1}\left(\lambda^{r_{1}}\right)\right)$ so that $F_{i}^{r_{i}}$ has not real positive eigenvalues (always possible from Lemma 4) and $\psi_{i}\left(\lambda^{r_{i}}\right)=c_{F_{i}}{ }^{r_{i}}\left(\lambda^{r_{i}}\right) \cdot H_{i}\left(\lambda^{r_{i}}\right)$, where $H_{i}$ is chosen using Lemma 2 and Comment 3 so that $\operatorname{Res}\left(p_{F_{j}}(\lambda),\left(c_{F_{i}} \cdot H_{i}\right)^{\left[\frac{q_{j}}{q_{i}}\right]}\left(\lambda^{r_{i}}\right)\right) \neq 0$. We note that in this case $H_{i}$ is assumed to have non-negative coefficients.

We show now that the polynomial $\Pi_{j}(\lambda)$ of the following form:

$$
\Pi_{j}(\lambda)=\psi_{j-1}^{\left[\frac{1}{q_{j-1}}\right]}\left(\lambda^{r_{j-1}}\right) \cdot \ldots \cdot \psi_{2}^{\left[\frac{1}{q_{2}}\right]}\left(\lambda^{r_{2}}\right) \cdot \psi_{1}^{\left[\frac{1}{q_{1}}\right]}(\lambda)
$$

satisfies the conditions of Lemma 5. First, notice that the integers $r_{i}$ are chosen in such a way that all polynomials $\psi_{i}$ have real coefficients (Lemma 4 was used to construct $\psi_{i}$ for even $q_{i}$ 's). Second, from Lemma 3 it follows $\forall k \in \mathbb{N}$ that:

$$
\Pi_{j}^{[k]}(\lambda)=\psi_{j-1}^{\left[\frac{k}{q_{j-1}}\right]}\left(\lambda^{r_{j-1}}\right) \cdot \ldots \cdot \psi_{2}^{\left[\frac{k}{q_{2}}\right]}\left(\lambda^{r_{2}}\right) \cdot \psi_{1}^{\left[\frac{1}{q_{1}}\right]}(\lambda)
$$

and hence we have that since by construction $\psi_{i}^{\left[\frac{q_{i}}{q_{i}}\right]}\left(F_{i}^{r_{i}}\right)=0, \forall i=1,2, \ldots, j-1$ that the first condition of Lemma 5 holds:

$$
\Pi_{j}^{\left[q_{i}\right]}\left(F_{i}\right)=\psi_{j-1}^{\left[\frac{q_{i}}{q_{j-1}}\right]}\left(F_{i}^{r_{j-1}}\right) \cdot \ldots \cdot \psi_{2}^{\left[\frac{q_{i}}{q_{2}}\right]}\left(F_{i}^{r_{2}}\right) \cdot \psi_{1}^{\left[\frac{q_{i}}{q_{1}}\right]}\left(F_{i}\right)=0, i=1,2, \ldots, j-1
$$

Moreover, notice from $(38)$ that $\Pi_{j}^{\left[q_{j}\right]}(\lambda)=\prod_{i=1}^{j-1} \psi_{i}^{\left[\frac{q_{j}}{q_{i}}\right]}\left(\lambda^{r_{i}}\right)$. It follows that $\operatorname{Res}\left(p_{F_{j}}(\lambda), \Pi_{j}^{\left[q_{j}\right]}(\lambda)\right) \neq 0$ if and only if $\operatorname{Res}\left(p_{F_{j}}(\lambda), \psi_{i}^{\left[\frac{q_{j}}{q_{i}}\right]}\left(\lambda^{r_{i}}\right)\right) \neq 0, \forall i=1,2, \ldots, j-1$, which holds by construction of the polynomials $\psi_{i}$. Hence, the second condition of Lemma 5 is also satisfied. Q.E.D. 
Notice that polynomials $\psi_{i}$ and integers $r_{i}$ in the proof of Lemma 5 are chosen in such a way that we can repeatedly use Lemma 2 for each of the polynomials $\psi_{i}$ and $p_{F_{j}}$ separately. We emphasize that other constructions are possible and the polynomial $\Pi_{r}(\lambda)$ constructed in Lemma 5 is obviously not unique. Also, the procedure which we presented does not yield the minimum degree of the polynomial.

Proof of Theorem 7: Necessity of the proof is obvious and we concentrate only on sufficiency. First, we can assume that all subsystems $S_{i}$ of the system (1) are completely controllable and the matrices $F_{i}$ are non-singular. Also, $q_{3}$ is assumed to be odd for simplicity.

Since system $S_{1}$ is null controllable we can assume that $x_{1}(0)=0$. Then, using the control sequences from the proof of Theorem 5 , it follows that we can zero $x_{2}$ while $x_{1}$ is also kept at zero. Hence, we assume that $x_{1}(0)=0$ and $x_{2}(0)=0$. If we consider the polynomial $\Pi_{3}(\lambda)$, which is constructed in Lemma 5 for pairs $\left(F_{1}, q_{1}\right),\left(F_{2}, q_{2}\right),\left(F_{3}, q_{3}\right)$, we have that $\Pi_{3}^{\left[q_{1}\right]}\left(F_{1}\right)=0$ and $\Pi_{3}^{\left[q_{2}\right]}\left(F_{2}\right)=0$. Apply now the control sequence $U[\Pi(\lambda)]$ defined in (26) with $q_{1}=1$, which yields:

$$
\begin{aligned}
& x_{1}(P)=F_{1}^{P} \underbrace{x_{1}(0)}_{=0}+\underbrace{\prod_{3}^{\left[q_{1}\right]}\left(F_{1}\right)}_{=0}\left[F_{1}^{k_{n_{3}-1}} g_{1}: \ldots: g_{1}\right]\left[v\left(n_{3}-1\right) \ldots v(0)\right]^{T}=0 \\
& x_{2}(P)=F_{2}^{P} \underbrace{x_{2}(0)}_{=0}+\underbrace{\prod_{3}^{\left[q_{2}\right]}\left(F_{2}\right)}_{=0}\left[F_{2}^{k_{n_{3}-1}} g_{2}: \ldots: g_{2}\right]\left[v^{2}\left(n_{3}-1\right) \ldots v^{2}(0)\right]^{T}=0 \\
& x_{3}(P)=F_{3}^{P} x_{3}(0)+\Pi_{3}^{\left[q_{3}\right]}\left(F_{3}\right)\left[F_{3}^{k_{n_{3}-1}} g_{3}: \ldots: g_{3}\right]\left[v^{3}\left(n_{3}-1\right) \ldots v^{3}(0)\right]^{T}
\end{aligned}
$$

From Lemma 1, it follows that we can find a sequence of integers $k_{i}, i=0, \ldots, n_{3}-1$ such that $F_{3}^{k_{i}} g_{3}$ span $\mathbb{R}^{n_{3}}$. Since the matrix $\Pi_{3}^{\left[q_{3}\right]}\left(F_{3}\right)$ is non-singular by construction of $\Pi_{3}$ in Lemma 5 , then for any $x_{3}(0)$ we can find $v(i)$ such that $x_{3}(P)=0$. Situations with more subsystems and/or $q_{3}$ even are tackled in a similar way using Lemmas $1-5$ and proof of Theorem 5 to construct the desired control sequences. They are omitted for space reasons. Q.E.D.

Comment 7 Although in the proof of our main results we did not work with the shortest control sequences which achieve dead-beat behavior, we have established that the null controllability property is with the uniform bound on the dead-beat time. In other words, if the system (1) is null controllable, then there is a fixed integer $\bar{N}$ such that any initial state can be transfered to the origin in at most $\bar{N}$ time steps.

\section{Continuous-time case}

In this section we show that the results on complete controllability of discrete-time systems can be used to prove an equivalent result for polynomial continuous-time systems. This is done by showing that a continuous-time system of the same structure as (1), whose subsystems are completely controllable, can be transformed into a discrete-time systems whose subsystems are completely controllable by using piecewise constant controls (sampler and zero order hold).

Before we state the main result of this section we need to state some preliminaries. We denote the control function restricted to the time interval $\left[t_{0}, t_{f}\right]$ as $u_{\left[t_{0}, t_{f}\right]}$. The state of the system (40) at time $T$, which emanates from the initial state $x(0)$ under the control $u_{[0, T]}$ is denoted as $x\left(T, x(0), u_{[0, T]}\right)$.

Definition 6 A continuous-time system $\Sigma$ is completely controllable if given any states $x(0), x_{f}$, there exists $T \in \mathbb{R}$ and control $u_{[0, T]}$ such that $x_{f}=x\left(T, x(0), u_{[0, T]}\right)$.

In this section we consider systems of the following form:

$$
\begin{aligned}
& S_{1}: \dot{x}_{1}(t)=A_{1} x_{1}(t)+b_{1} u^{q_{1}}(t) \\
& S_{2}: \dot{x}_{2}(t)=A_{2} x_{2}(t)+b_{2} u^{q_{2}}(t) \\
& \text {... } \quad \ldots \\
& S_{m}: \dot{x}_{m}(t)=A_{m} x_{m}(t)+b_{m} u^{q_{m}}(t)
\end{aligned}
$$

where $x_{i} \in \mathbb{R}^{n_{i}}, i=1,2, \ldots, m, u(t) \in \mathbb{R}$ and matrices $A_{i}, b_{i}$ have the appropriate dimensions.

We remark that conditions for complete controllability of subsystems $S_{i}$ of the continuous-time system (40) are known from the literature:

Theorem 9 A subsystem $S_{i}$ of (40) is completely controllable if and only if: 
1. $[K a]$ for $q_{i}=2 t+1, t \in \mathbb{N}$

(a) $\operatorname{rank}\left[\lambda I-A_{i}: b_{i}\right]=n_{i}, \forall \lambda \in \mathbb{C}$

2. $[S a]$ for $q_{i}=2 t, t>0, t \in \mathbb{N}$

(a) $\operatorname{rank}\left[\lambda I-A_{i}: b_{i}\right]=n_{i}, \forall \lambda \in \mathbb{C}$

(b) $A_{i}$ has no real eigenvalues

The main result of this section is given below:

Theorem 10 The system (40) is completely controllable if and only if all of its subsystems $S_{i}, i=$ $1,2, \ldots, m$ are completely controllable.

From Theorems 9 and 10 we can see that the test for complete controllability of the system (40) is again very easy-to-check and consists of $m$ controllability tests for its subsystems $S_{i}$.

Proof of Theorem 10: Necessity of the proof is trivial and we concentrate only on the sufficiency.

Assume that the control signal is piecewise constant, that is:

$$
u(t)=u(k)=\text { const., } \forall t \in[k h,(k+1) h[, h>0, k \in \mathbb{N}
$$

In other words, we assume that a zero order hold and sampler are used. The particular structure of the system (40) allows us to obtain a discrete-time model of the system in the same manner as for the linear systems. Indeed, the discrete-time model of the system (40) with the assumption of zero order hold, is given by (1), where

$$
F_{i}=e^{A_{i} h}, \quad g_{i}=\int_{0}^{h} e^{A_{i} s} b_{i} d s
$$

If we can find a sampling period $h$ such that for all controllable subsystems $S_{i}$ of (40), we obtain that all the subsystems of the system (1) with (41) are controllable, the proof of Theorem 10 follows immediately from Theorem 7.

All subsystems $S_{i}$ in (40) are controllable. Consider the subsystem $S_{i}$ for which $q_{i}$ is odd. Denote the eigenvalues of the matrix $A_{i}$ as $\sigma_{t}=\rho_{t}+j \omega_{t}$. From [Ka][pg. 174-175] it follows that since the pair $\left(A_{i}, b_{i}\right)$ is controllable, the pair $\left(F_{i}, g_{i}\right)$, which is computed using $(41)$, will be controllable if and only if whenever

$$
\rho_{i}-\rho_{l}=0 \text { then } h \neq \frac{2 k \pi}{\omega_{i}-\omega_{l}}, \quad i, l \in\left\{1,2, \ldots, n_{i}\right\}, k \in \mathbb{N}
$$

Hence only countably many values of $h$ are critical. That is, the discrete-time subsystem $S_{i}$ may not be controllable only for the values of $h$ defined in (42).

On the other hand, if we consider a subsystem $S_{i}$ for which $q_{i}$ is even, we need besides the controllability condition of the pair $\left(F_{i}, g_{i}\right)$ also that $F_{i}$ has no positive real eigenvalues. Notice that if the matrix $A_{i}$ had any real eigenvalues (the continuous-time subsystem $S_{i}$ is not controllable), then for any sampling period $h>0$, the matrix $F_{i}$ defined by (41) would have a real positive eigenvalue, and hence the discretized subsystem is also not controllable. Since the matrix $A_{i}$ has only complex eigenvalues $\sigma_{t}=\rho_{t}+j \omega_{t}, \omega_{t} \neq 0, \forall t$, if the sampling period is chosen so that:

$$
h \neq \frac{2 k \pi}{\omega_{t}}, \forall t \in\left\{1,2, \ldots, n_{i}\right\}, k \in \mathbb{N}
$$

then $F_{i}$ has no positive real eigenvalues. In summary, the critical values of $h$ are given by conditions (42) and (43) and therefore it is always possible to choose $h>0$ so that all subsystems of the discretized system (1) with (41) are controllable. Notice also that $F_{i}$ obtained using (41) are all non-singular. The proof of Theorem 10 follows from Corollary 3. Q.E.D. 


\section{Summary}

We have presented natural and easy-to-check necessary and sufficient conditions for null and complete controllability for a class of discrete-time polynomial systems. Interesting relationships and differences between the present class of systems, purely linear systems and generalized Hammerstein systems considered in [Ne1] were commented on. Our results allow for an interesting interpretation which says that by "non-linearizing" an uncontrollable linear system we may recover controllability. The result for discrete-time systems was used to prove a continuous-time controllability result.

Acknowledgments: The author wishes to thank anonymous reviewers for pointing out an error in the original proof of Lemma 2 and giving lots of constructive comments which improved the presentation of the paper. This work was supported by the Australian Research Council under the ARC Large Grant Scheme.

\section{References}

[BaIs] W. Bamberger and R. Isermann, Adaptive on-line steady-state optimization of slow dynamic processes, Automatica 14 (1978), 223-230.

[CLO] D. Cox, J. Little and D. O'Shea, Ideals, varieties, and algorithms: an introduction to computational algebraic geometry and commutative algebra, Springer-Verlag, New York, 1992.

[EvMu1] M. E. Evans and D. N. P. Murthy, Controllability of a class of discrete-time bilinear systems, IEEE Trans. Automat. Control, 22 (1977), 78-83.

[EvMu2] M. E. Evans and D. N. P. Murthy, Controllability of discrete-time systems with positive controls, IEEE Trans. Automat. Control, 22 (1977), 942-945.

[EvMu3] M. E. Evans and D. N. P. Murthy, Controllability of discrete-time inhomogeneous bilinear systems, Automatica, 14 (1978), 147-151.

[Ev] M. E. Evans, The convex controller: controllability in finite time, Int. J. Sys. Science, 16 (1985), 31-47.

[HaKe] R. Haber and L. Keviczky, Identification of nonlinear dynamic systems, in: N. S. Rajbman (ed.), Identification and system parameter estimation, North-Holland, 1978, 79-127.

[HaUn] R. Haber and H. Unbehauen, Structure identification of nonlinear dynamic systems-a survey of input/output approaches, Automatica 26 (1990), 651-677.

[Ka] T. Kailath, Linear systems, Prentice Hall, New Jersey, 1980.

[Ke $]$ L. Keviczky, Nonlinear dynamic identification of a cement mill to be optimized, in: N. S. Rajbman (ed.), Identification and system parameter estimation, North-Holland, 1978, 11851195.

[NMBM1] D. Nešić, I. M. Y. Mareels, R. Mahony and G. Bastin, $\nu$-step controllability of scalar polynomial systems, Proc. 3rd ECC, Rome, Italy, 1995, 277-282.

[NMBM2] D. Nešić, I. M. Y. Mareels, G. Bastin and R. Mahony, Necessary and sufficient conditions for output dead beat controllability for a class of polynomial systems, Proc. IEEE CDC, New Orleans, 1995, 7-13.

[NeMa1] D. Nešić and I. M. Y. Mareels, Invariant sets and output dead beat controllability for odd polynomial systems: the Gröbner basis method, Proc. 13th IFAC World Congress, San Francisco, vol. E, 1996, 221-226.

[Ne1] D. Nešić, A note on null controllability of generalized Hammerstein models, Sys. Contr. Lett, 29 (1997), 223-231.

[Ne2] D. Nešić, A note on observability for general polynomial and simple Wiener-Hammerstein systems, Sys. Contr. Lett., 35 (1998), 219-227. 
[Ne3] D. Nešić, Controllability for a class of simple Wiener-Hammerstein systems, to appear in Sys. Contr. Lett.

[NeMa2] D. Nešić and I. M. Y. Mareels, Dead-beat controllability of polynomial systems: symbolic computation approaches, IEEE Trans. Automat. Contr., 43 (1998), 162-175.

[NMBM3] D. Nešić, I. M. Y. Mareels, G. Bastin and R. Mahony, Output dead-beat control for a class of planar polynomial systems, SIAM J. Contr. Optimiz., 36 (1998), 253-272.

[ORe] J. O'Reilly, The linear time invariant time-optimal control problem - an overview, Automatica, 17 (1981), 363-370.

[Sa] S. H. Saperstone, Global controllability of linear systems with positive controls, SIAM J. Contr., 11 (1973), 417-423.

[So] E. D. Sontag, Controllability is harder to decide than accessibility, SIAM J. Contr. Optimiz., 26 (1988), 1106-1118. 